\title{
Linear and nonlinear buckling analysis of a locally stretched plate
}

\author{
Madina Kilardj ${ }^{1}$, Ghania Ikhenazen ${ }^{1}$, Tanguy Messager ${ }^{2}$ and Toufik Kanit ${ }^{2}$ \\ ${ }^{1}$ University of Sciences and Technology Houari Boumediene (U.S.T.H.B), El Alia 16111 Bab Ezzouar, Algiers, Algeria \\ ${ }^{2}$ Laboratoire de Mécanique de Lille (UMR CNRS 8107), Université Lille 1, Cité Scientifique, 59655 Villeneuve d'Ascq cedex, France
}

\begin{abstract}
Uniformly stretched thin plates do not buckle unless they are in special boundary conditions. However, buckling commonly occurs around discontinuities, such as cracks, cuts, narrow slits, holes, and different openings, of such plates. This study aims to show that buck-ling can also occur in thin plates that contain no defect or singularity when the stretching is local. This specific stability problem is ana-lyzed with the finite element method. A brief literature review on stretched plates is presented. Linear and nonlinear buckling stress analyses are conducted for a partially stretched rectangular plate, and various load cases are considered to investigate the influence of the partial loading expanse on the critical tensile buckling load. Results are summarized in iso-stress areas, tables and graphs. Local stretch-ing on one end of the plate induces buckling in the thin plate even without geometrical imperfection.
\end{abstract}

Keywords: Buckling; FEM; Linear and nonlinear analysis; Local stretching; Thin plate

\section{Introduction}

Thin plates are structural elements that are widely used in many engineering fields, such as aerospace, shipbuilding and civil engineering. Ribbed and stiffened ship plate, offshore, and aerospace panels are commonly utilized and always subjected to partial edge traction on their own plane. This type of loading can induce buckling, which negatively affects the function of the structural elements concerned.

Plate instability is generated globally or locally through inplane compression and shear loading. The corrugations that result from plate instability may cause permanent deformation and reduce the efficiency of the entire structure. Thin plate buckling under compression and shear was studied by Timoshenko [1] and Bulson [2], and analytical solutions were presented. Chen and Kim [3] utilized a combined equivalent energy method and proposed a stretched corrugated plate model. Numerical methods, such as finite element method, can solve particular structures (e.g., crack propagation in a bearing ring [4], blast loaded corroded plates [5] and stretched corrugated beam elements [6]) and have provided solutions to highly complex problems encountered in plate buckling, such as laminated composite plates under compression [7], partially compressed plate [8], and defective plate under shear [9], uniform compression $[10,11]$ and local compression [12].

The buckling phenomenon appears as a result of the appli- cation of compression or shear load; it occurs in less usual situations, in which tensile loading induces buckling instability and the formation of buckling configurations. Despite the fact that no compressive component exists in the applied tension, local compressive stresses resulting from the presence of a cut, crack, or hole induce stability loss.

Datta and Carlson [13] presented the results of an analytical and experimental study on the buckling of a thin sheet with a hole in the tensile field. The analytical method used in stress distribution around the opening was based on the elastic solution introduced by Timoshenko and Goodier [14].

Shaw [15] and Brighenti [16-18] utilized the finite element method to investigate the buckling of a defective uniformly tensioned plate. A few authors studied the effect of point loading. Tomita et al. [19], Woo and Jenkins [20], and Kremer [21] examined the case of concentrated tensile load applied to defective thin plates.

The experimental studies of Gilabert et al. [22] and Seifi [23] also revealed that local buckling occurs around defects on a stretched plate. The waves of the buckling undulation are perpendicular to the applied tensile stress.

These studies have shown that perfectly flat plates subjected to tensile load may buckle locally in the presence of defects. However, buckling occurs even in the absence of any geometric discontinuity. Such a phenomenon was well described by Bryan [24], who compared a tensioned plate in one direction to a stretched sheet of paper wet in the middle. Moisture, which illustrates paper elasticity rather than weakness, causes the surface of the paper to expend and wrinkle. The wrinkles 
become fine and close as the tensile forces increase. This issue was discussed by Bryan [24] through an analytic method. Friedl et al. [25] presented a numerical solution to this problem for special boundary conditions. However, only a few studies have been conducted on partially tensioned plates that exhibit no geometric discontinuity or imperfection. Moreover, only a few authors have addressed the problem of nonlinear buckling of thin stretched plates; even when an analysis on such plates had been conducted, the partially tensioned plate was not considered.

In the present study, the linear and nonlinear buckling of a thin, partially stretched, non-defective plate at its one end in the lateral direction, as depicted in Fig. 1, was investigated with the Finite element method (FEM). The aim of this study is to provide representative linear and nonlinear buckling load results for a simply supported plate. The effect of applied load length and plate length $(l / b)$ ratio on the critical buckling load was investigated. The obtained numerical results in both linear and nonlinear fields were graphically summarized, and several interesting conclusions were obtained. To the authors' best knowledge, no experimental study is currently available to validate the results presented in this numerical investigation.

\section{Stability problem formulation}

Analytical estimations should be performed prior to numerical buckling analyses. However, no analytical solution exists for the present extremely complex buckling problem. The governing differential equation for the buckled plate is [1]

$$
\frac{\partial^{4} w}{\partial x^{4}}+2 \frac{\partial^{4} w}{\partial x^{2} \partial y^{2}}+\frac{\partial^{4} w}{\partial y^{4}}=\frac{1}{D}\left(N_{x} \frac{\partial^{2} w}{\partial x^{2}}\right)
$$

where $w$ is the plate deflection and $D$ is the plate flexural rigidity. An analogous problem with a uniformly distributed compressive load applied over the width $b$ of a simply supported thin plate was treated analytically in a previous study and has led to the well-known expression of the compressive load critical value as follows [1]:

$$
N_{x}=k \frac{\pi^{2} D}{b^{2}} \text { for } a / b \geq 1
$$

where $k$ is the plate buckling coefficient. However, Eq. (2) is inapplicable to the case of patch tensile loading, and numerical methods, such as FEM, should be utilized. The linear tensile buckling problem is formulated as an eigenvalue problem leading to the equation

$$
\left([K]+\lambda_{i}[S]\right)\{\Psi\}_{i}=\{0\}
$$

\footnotetext{
where

$[K]$ : Stiffness matrix,

$[S]$ : Stress stiffness matrix,
}

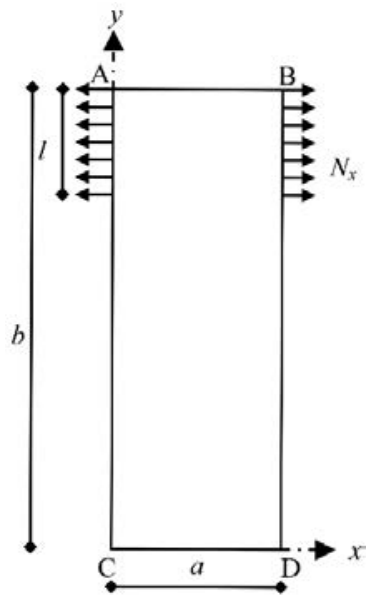

Fig. 1. Partially stretched thin plate.

$\lambda_{i}$ : ith eigenvalue used to multiply the loads that are generated $[S]$, and

$\Psi_{i}$ : ith eigenvector of displacements.

Hook's law is used to relate the strain vector $\{\varepsilon\}$ to the resultant stress vector $\{\sigma\}$.

$$
\{\sigma\}=[D] \times\{\varepsilon\},
$$

with: $\{\sigma\}=\left\lfloor\sigma_{x} \sigma_{y} \sigma_{z} \sigma_{x y} \sigma_{y z} \sigma_{x z}\right\rfloor^{T}$;

$$
\{\varepsilon\}=\left\lfloor\varepsilon_{x} \varepsilon_{y} \varepsilon_{z} \varepsilon_{x y} \varepsilon_{y z} \varepsilon_{x z}\right\rfloor^{T}
$$

The Von-Mises' stress is computed as

$$
\sigma_{\text {Von-Mises }}=\left(\frac{1}{2}\left[\left(\sigma_{x}-\sigma_{y}\right)^{2}+\left(\sigma_{y}-\sigma_{z}\right)^{2}+\left(\sigma_{z}-\sigma_{x}\right)^{2}+6\left(\sigma_{x y}^{2}+\sigma_{y z}^{2}+\sigma_{x z}^{2}\right)\right]\right)^{\frac{1}{2}} .
$$

\section{Results and discussions}

\subsection{Description and modelling of the analyzed plate}

\subsubsection{Description of the analyzed plate}

The present investigation aims to examine the buckling behavior of a mild steel plate subjected to localized lateral tension (see Fig. 1). The plate is simply supported at its short sides and free at its lateral loaded edges. Only the displacement in the $\mathrm{z}$ direction is prevented on the $\mathrm{AB}$ side (Fig. 1), whereas the prevented displacements on the opposite side, $\mathrm{CD}$, are along the $\mathrm{z}$ and $\mathrm{y}$ axes. A middle node is also restrained in the $\mathrm{x}$ direction on this last edge. Aspect ratio $a / b$, where $a$ and $b$ are the width and length of the examined plate, respectively, is equal to $1 / 3$. Aspect ratio $l / b$, where $l$ is the distance over which the localized load extends on the plate length, provides different load cases to be analyzed. Given the importance of the plate thickness effect on the buckling load, the $a / h$ ratio, where $h$ is the plate thickness, is equal to 100 . Poisson's ratio $v=0.3$, Young modulus $E=2.09 \times 10^{5} \mathrm{MPa}$, 
Table 1. Critical linear buckling loads obtained in the present convergence analysis using (a), (b) and (c) meshes for different loading cases.

\begin{tabular}{c|c|c|c|c|c}
\hline \multirow{2}{*}{$l / b$} & \multicolumn{3}{|c|}{$\mathrm{P}_{\text {cr }}[M N / m l]$} & \multicolumn{2}{c}{ Error rate $[\%]$} \\
\cline { 2 - 6 } & (a) & (b) & (c) & (b) & (c) \\
\hline 0.1667 & 7.7810 & 7.7820 & 7.7815 & $10^{-2}$ & $10^{-2}$ \\
\hline 0.3333 & 8.4427 & 8.4460 & 8.4407 & $4 \times 10^{-2}$ & $2 \times 10^{-2}$ \\
\hline 0.5000 & 8.4793 & 8.4783 & 8.4781 & $10^{-2}$ & $10^{-2}$ \\
\hline 0.6667 & 8.8732 & 8.8724 & 8.8740 & $10^{-2}$ & $10^{-2}$ \\
\hline 0.8333 & 9.9793 & 9.9791 & 9.9789 & 0 & 0 \\
\hline
\end{tabular}

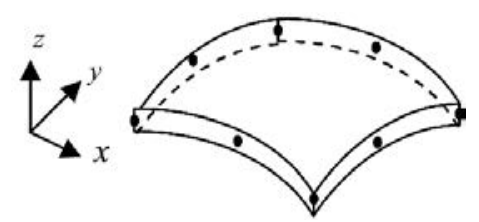

Fig. 2. Isoparametric quadrilateral element with eight nodes.

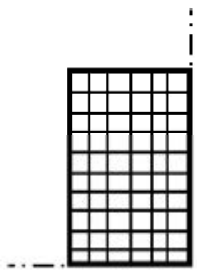

(a)

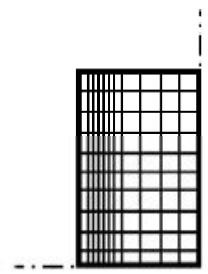

(b)

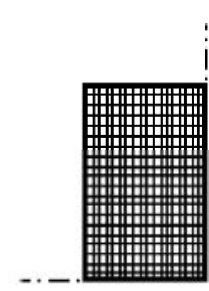

(c)
Fig. 3. Meshes studied in the present convergence analysis.

and yielding strength $\sigma_{\text {vield }}=400 \mathrm{MPa}$ were also considered.

\subsubsection{Finite element mesh density}

The plate in the numerical tests performed with ANSYS 14.5 software academic version was modeled by an eightnodded element (depicted in Fig. 2). This element with six degrees of freedom at each node is well-suitable for linear and nonlinear applications.

To obtain reasonable results, several successive linear analyses were conducted to determine the optimum mesh density to be used. Three different types of meshes were designed for the plate described in Sec. 3.1.1 to perform a convergence analysis. The grids are depicted in Fig. 3 by their quarters. The Fig. 3(a) type mesh is uniformly gridded with $50 \times 150$ elements. The Fig. 3(b) type mesh that contains $70 \times 150$ elements is refined only at the neighborhood of the loaded edge. The Fig. 3(c) type mesh comprising $100 \times 300$ elements is uniformly and tightly refined. The subdivisions refer to the entire plate in the $\mathrm{x}$ and $\mathrm{y}$ directions. Table 1 presents a summary of the results obtained in the conducted numerical experiments. The figures in Table 1 show that the use of a mesh particularly refined in the vicinity of the local stretching (b) or entirely and tightly gridded (c) does not cause important changes in the results. Similar results were obtained by Deolassi [26]. Consequently, the mapped discretization of type (a) depicted in Fig. 3(a) was adopted in the buckling analysis.

\subsection{Linear and nonlinear plate buckling analyses}

The numerical experiments show that the examined plates in their different mode shapes always buckle in the direction perpendicular to the applied tensile load. Thus, only stresses in this direction were analyzed. Several quantitative results were recorded for the plate described in Sec. 3.1.1 by adopting the ratio $l / b=1 / 6$; these results are presented in Table 2 .

The finite element results suggest that a limited number of mode shapes is sufficient to describe the plate buckling behavior correctly [8]. The first 50 mode shapes were derived, and the first 5 mode shapes in the linear and nonlinear fields were recorded and are shown in Fig. 4.

\subsubsection{Linear longitudinal stress analysis}

Fig. 4 shows the iso-stress areas for the linear longitudinal stress component on the entire plate. The drawing in the figure shows that negative stresses, which are compressive stresses perpendicular to the direction of the applied tensile loading, occurred in different parts of the plate. These stresses are evidently responsible for the occurrence of plate buckling because of the Poisson's effect, which is associated with the present boundary conditions. The maximum longitudinal stresses, both compressive and tensile, are located on the plate edges where in-plane loading was directly applied. The stress value decreased toward the longitudinal center line region and eventually reached its minimum. The maximum compressive stress showed a significant value of $20 \%$ higher than the maximum tensile stress although the loading was tensile (see Table 2). However, the minimum compressive stress value was almost nine times greater than the minimum tensile stress value. Fig. 5 shows that compressive stress covers the plate surface more than tensile stress does. The compressive zone is about five times larger than the tensile zone.

\subsubsection{Nonlinear longitudinal stress analysis}

The described plate was also analyzed in the nonlinear field. Fig. 6 shows the iso-stress areas for the nonlinear longitudinal stress component on the entire plate. The iso-stress areas show that compressive stresses perpendicular to the direction of the applied tensile loading occurred but over a much smaller surface area. The obtained nonlinear values show that the compressive stresses are exclusively located on the plate edge and in its nearby neighborhood, whereas the tensile stress spreads all over the plate and reaches its maximal value in the centerline region in the applied load vicinity.

The maximum compressive stress shows a significant value of 5.5 times the maximum tensile stress. Moreover, when the maximum compressive stress value in the linear analysis is smaller than that obtained in the nonlinear analysis, the maximum tensile stress value is greater than that obtained in the nonlinear field. The maximum stress values in both analyses 
Table 2. Maximal and minimal buckling stress values ( $\sigma_{c}=$ compressive stress value, $\sigma_{t}=$ tensile stress value) in the present work.

\begin{tabular}{c|c|c|c|c|c|c}
\hline-- & $\sigma_{c \max }[\mathrm{MPa}]$ & $\sigma_{c \min }[\mathrm{MPa}]$ & $\sigma_{t \max }[\mathrm{MPa}]$ & $\sigma_{t_{\min }}[\mathrm{MPa}]$ & $\sigma_{c \max } / \sigma_{t \max }$ & $\sigma_{c \min } / \sigma_{t \min }$ \\
\hline Linear analysis & -210.6000 & -38.6382 & 176.3140 & 4.3522 & 1.194 & 8.878 \\
\hline Nonlinear analysis & -351.9490 & -28.4486 & 63.9802 & 17.7658 & 5.501 & 1.601 \\
\hline
\end{tabular}
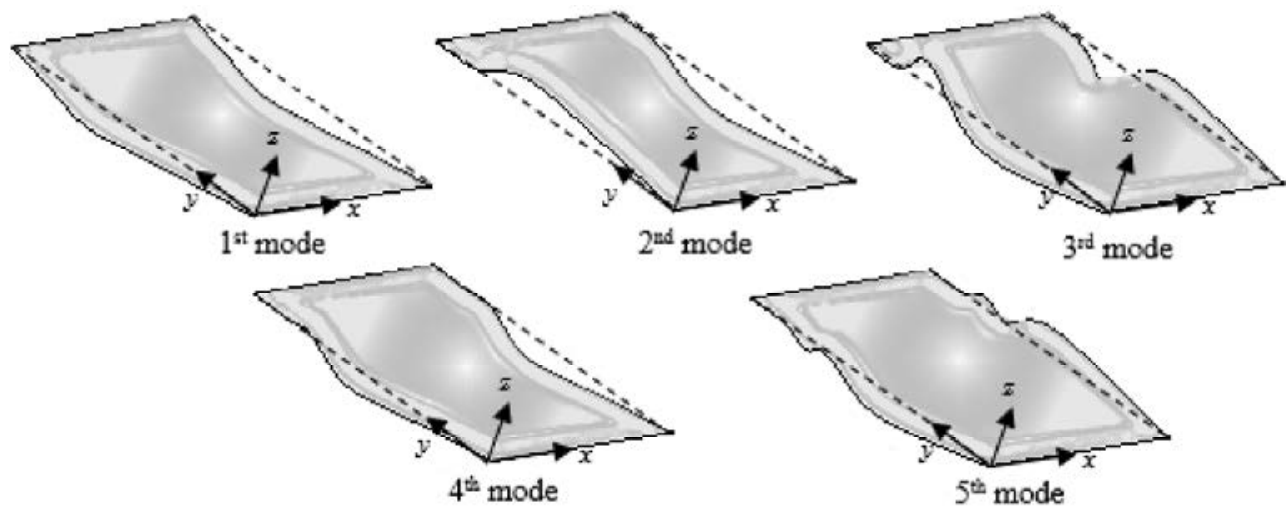

Fig. 4. Buckling mode shape configurations obtained in the present investigation for a plate with $a / b=1 / 3$ and $l / b=1 / 6$.

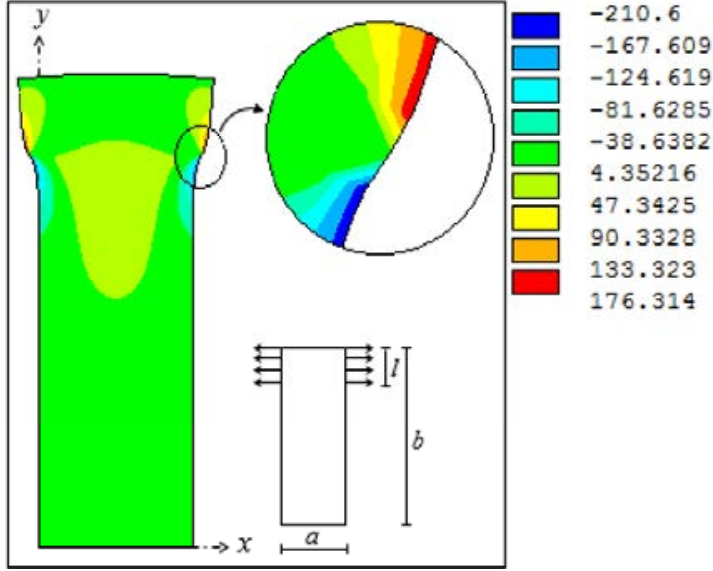

Fig. 5. Linear iso-stress areas (in $\mathrm{MPa}$ ) for $y$ direction stresses on a plate with $a / b=1 / 3$ and $l / b=1 / 6$.

are less than the yielding strength. Therefore, plate instability occurred in the elastic field.

\subsubsection{Von-Mises' stress analysis}

A Von-Mises' stress analysis in both linear and nonlinear fields was performed to provide further insight into the elastoplastic behavior of the plate. The obtained results are presented in Figs. 7 and 8.

Comparison of the two stress distributions reveals that in the nonlinear analysis, plasticization occurred in the area bounded by the loading application points and the width of the simply supported plate. However, in the linear analysis where the obtained failure area is much smaller, this phenomenon appeared partially in the loaded zone. Furthermore, in the linear field, failure was reached with the stress value varying between $6 \%$ and $20 \%$ of the yielding strength, whereas in the

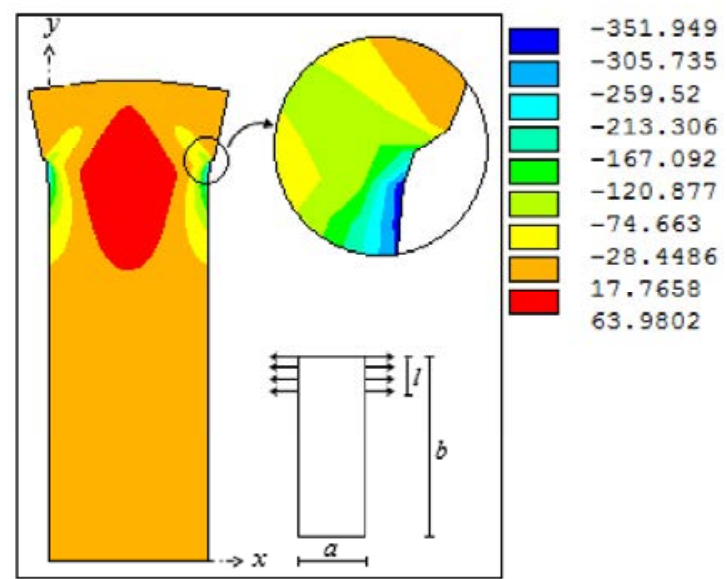

Fig. 6. Nonlinear iso-stress areas (in $\mathrm{MPa}$ ) for $y$ direction stresses on a plate with $a / b=1 / 3$ and $l / b=1 / 6$.

nonlinear field, failure was obtained with a stress value equal to the yielding stress. Yielding may not occur in this last plate configuration. Thus, the strength of the whole material is utilized to the best advantage.

\subsection{Critical buckling load for different load cases}

The previous described plate was studied for different load cases to investigate the influence of partial loading expanse on critical tensile buckling load $P_{c r}$. The $l / b$ ratio value, which is the ratio between the distance over which the tensile loading extends and the plate length, was varied from 0.033 to 1.000 with an increase of one-thirtieth in each numerical test. Fig. 9 shows critical tensile buckling load $P_{c r}$ versus the $l / b$ ratio. Given that it is used in plate stability, the critical buckling load resulting from numerous localized tensile load 
Table 3. Critical resultant buckling load $R_{c r}$ values reached in the present investigation for $0 \leq l / b \leq 0.20$.

\begin{tabular}{|c|c|c|c|c|c|c|}
\hline \multirow{2}{*}{$l / b$} & \multicolumn{2}{|c|}{$P_{c r}[M N / m l]$} & \multicolumn{2}{|c|}{$R_{c r}[M N]$} & \multicolumn{2}{|c|}{$R_{c r}$ relative increase } \\
\hline & Linear analysis & Nonlinear analysis & Linear analysis & Nonlinear analysis & Linear analysis & Nonlinear analysis \\
\hline 0 & \multicolumn{2}{|c|}{0.41} & \multicolumn{2}{|c|}{0.41} & \multicolumn{2}{|c|}{---} \\
\hline 0.03 & \multicolumn{2}{|c|}{7.82} & \multicolumn{2}{|c|}{0.78} & \multicolumn{2}{|c|}{$1.90 \cong 2 P_{c r}(l / b=0)$} \\
\hline 0.07 & 6.34 & 6.00 & 1.27 & 1.20 & $3.10 \cong 3 P_{c r}(l / b=0)$ & $2.93 \cong 3 P_{c r}(l / b=0)$ \\
\hline 0.20 & 8.14 & 4.69 & 4.88 & 2.81 & --- & $6.85 \cong 7 P_{c r}(l / b=0)$ \\
\hline
\end{tabular}

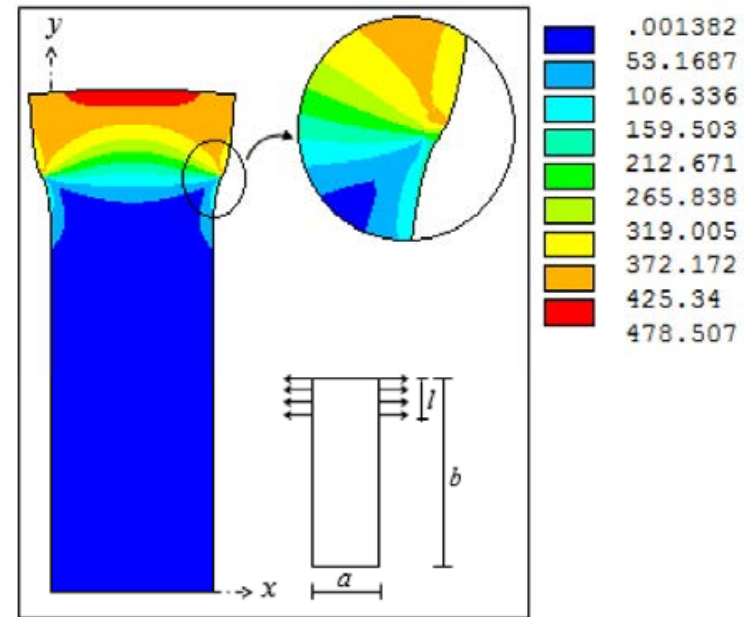

Fig. 7. Linear Von-Mises' iso-stress areas (in MPa) on a plate with $a / b=1 / 3$ and $l / b=1 / 6$.

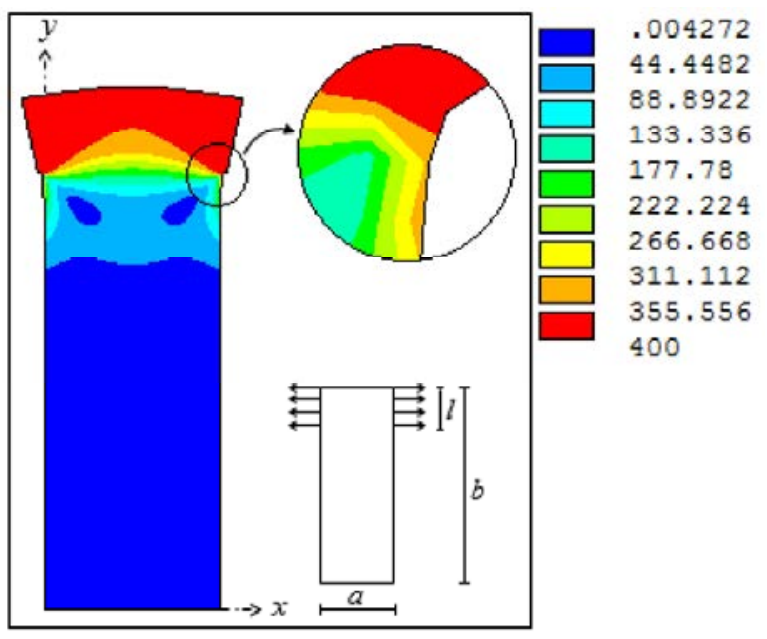

Fig. 8. Nonlinear Von-Mises' iso-stress areas (in MPa) on a plate with $a / b=1 / 3$ and $l / b=1 / 6$.

cases may be derived from this graph for a simply supported plate with $a / b=1 / 3$. A change of scale for $l / b$ values up to 0.200 is shown in Fig. 9. The continuous line represents the results of linear buckling analysis, and the dashed line represents those of nonlinear analysis.

Fig. 9 shows that in the linear and nonlinear analyses, the

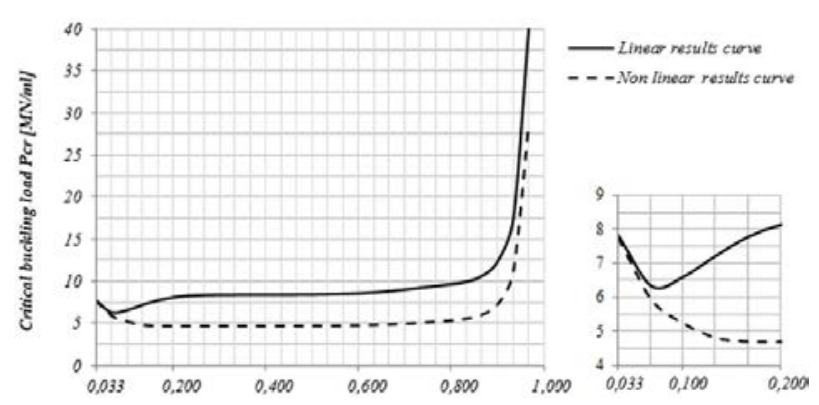

Fig. 9. Critical buckling load $P_{c r}$ versus $l / b$ ratio for a plate with $a / b=1 / 3$.

graphs show the same global tendency, but linear analysis generally has a larger $P_{c r}$ value. Furthermore, in the numerical tests conducted for $l / b=1$, the plate shows no buckling when the loading is uniform. The obtained critical buckling load value is $P_{c r}(l / b=0)=0.41 M N$ in both linear and nonlinear fields when loading is concentrated on one unique point.

Comparison of the two curves obtained in the linear and nonlinear buckling analyses shows three common significant sections as follows.

(1) The first section is delimited by $0.033 \leq l / b \leq 0.200$ values. The nonlinear curve reduces the overlap in the linear one up to about $l / b=0.067$. The linear curve increases when $0.067 \leq l / b \leq 0.200$. Given that $l / b$ is small in this section, critical loading $P_{c r}$ can be likened to concentrated load, the value of which is the resultant loading $R_{c r}=l \times P_{c r}$. Table 3 shows several $R_{c r}$ derived values obtained in the linear and nonlinear analyses. The figures in Table 3 show that when $l / b \leq 0.067, R_{c r}$ increases almost proportionally to $l / b$, and the nonlinear $R_{c r}$ value maintains this behavior up to $l / b=0.200$. The resultant linear buckling load increase is important for the last $l / b$ ratio. It is about 12 times as large as $P_{c r}(l / b=0)$. Moreover, the last linear $R_{c r}$ value reached is $74 \%$ greater than the corresponding nonlinear critical resultant buckling load. These results reveal the significant effect of material elasticity on plate behavior for small $l / b$ values.

(2) The second section, which is delimited by $0.200 \leq l / b \leq 0.900$ values, shows a constant curve behavior. Buckling occurs under the same critical load value regardless of the $l / b$ increase. Linear analysis in this section leads to a 
Table 4. Nonlinear to linear $P_{c r}$ rate values reached in the present investigation for $0.20 \leq l / b \leq 0.97$.

\begin{tabular}{c|c|c|c}
\hline \multirow{2}{*}{$l / b$} & \multicolumn{2}{|c|}{$P_{c r}[M N / m l]$} & Nonlinear to linear $P_{c r}$ \\
\cline { 2 - 3 } & $\begin{array}{c}\text { Linear } \\
\text { analysis }\end{array}$ & $\begin{array}{c}\text { Nonlinear } \\
\text { analysis }\end{array}$ & rate $[\%]$ \\
\hline 0.20 & 8.14 & 4.69 & 58 \\
\hline 0.90 & 12.50 & 7.50 & 60 \\
\hline 0.97 & 40.13 & 28.80 & 72 \\
\hline
\end{tabular}

critical buckling load of about $8.14 \mathrm{MN} / \mathrm{ml}$, whereas nonlinear analysis results in $P_{c r}$ with an average value of 4.69 MN / $\mathrm{ml}$, which is the minimum critical nonlinear buckling load.

(3) With $0.900 \leq l / b \leq 0.967$, both curves in the last section exhibit a dramatic increase in tensile buckling load with a small increase in the $l / b$ ratio. For $l / b=0.967$ in nonlinear analysis, $P_{c r}=28.80 \mathrm{MN} / \mathrm{ml}$; in linear analysis, $P_{c r}$ reaches the value of $40.13 \mathrm{MN} / \mathrm{ml}$.

The obtained nonlinear values are always lower than the linear results and are closer to those obtained from linear analysis because the loading tends to cover the entire plate length. This tendency is illustrated in Table 4 , which contains the $P_{c r}$ rates reached in the present investigation. Nonlinearities tend to prevent "ideal" structural elements from achieving their theoretical elastic buckling strength. Thus, the eigenvalue buckling load is somewhat overestimated. The numerical tests conducted in this study show that when the analyzed plates tended to be uniformly stretched, they developed material nonlinearities.

\section{Conclusions}

The present paper considered the buckling phenomenon of thin, simply supported, partially stretched plates. Numerical FEM analyses were performed to examine the buckled plate compressive and tensile stresses and determine the critical buckling loads for various load cases from uniform to point loading tension in linear and nonlinear domains.

The main purpose of the present study was achieved. Local tensile loading induces buckling instability in thin plates with no defects. Owing to the Poisson's effect, which is associated with the present boundary conditions, compressive stresses are obviously responsible for the occurrence of flat plate buckling.

In determining the plate buckling shapes, the direction of the buckling shape half waves is always perpendicular to that of the applied tensile load, and no buckling exists under uniform tension.

The linear and nonlinear numerical analyses showed that plate instability in specific boundary conditions occurs in the elastic field. Furthermore, the Von-Mises' nonlinear analysis showed that yielding may not occur, and the strength of the entire material is used to the best advantage, which is not the case in the linear analysis.
Finally, diagrams with linear and nonlinear critical buckling loads as a function of the ratio $l / b$ were introduced. The diagrams allow for the direct reading of the tensile critical load values for a plate with a ratio of $a / b=1 / 3$ for conventional plate buckling problems. These diagrams show the same global tendency; generally, in the linear analysis, a greater $P_{c r}$ value is obtained, and no buckling occurs when the loading is uniform.

Further research is required to investigate the experimental buckling load of partially stretched plates so that the present numerical results can be compared with those from experimental work.

\section{References}

[1] S. P. Timoshenko and J. M. Gere, Theory of elastic stability, McGraw-Hill, New York (1961).

[2] P. S. Bulson, The Stability of Flat Plates, Chatto and Windus, London (UK) (1970).

[3] Y. J. Chen and H. G. Kim, An equivalent plate model for corrugated-core sandwich panels, Journal of Mechanical Science and Technology, 29 (3) (2015) 1217-1223.

[4] S. Deng, X. Qin and S. Huang, A study on the effect of subsurface crack propagation on rolling contact fatigue in a bearing ring, Journal of Mechanical Science and Technology, 29 (3) (2015) 1029-1038.

[5] A. Eslami-majd and A. Rahbar-Ranji, Blast response of corroded steel plates, Journal of Mechanical Science and Technology, 28 (5) (2014) 1683-1690.

[6] P. Cartraud and T. Messager, Computational homogenization of periodic beam-like structures, International Journal of Solids and Structures, 43 (3) (2006) 686-696.

[7] A. Ghorbanpour Arani, Sh. Maghamikia, M. Mohammadimehr and A. Arefmanesh, Buckling analysis of laminated composite rectangular plates reinforced by swcnts using analytical and finite element methods, Journal of Mechanical Science and Technology, 25 (3) (2011) 809-820.

[8] G. Ikhenazen, M. Saidani and A. Chelghoum, Finite element analysis of linear plates buckling under in-plane patch loading, Journal of Constructional Steel Research, 66 (8) (2010) 1112-1117.

[9] R. Brighenti and A. Carpinteri, Buckling and fracture behavior of cracked thin plates under shear loading, Materials \& Design, 32 (3) (2011) 1347-1355.

[10] A. L. Narayana, K. Rao and R. V. Kumar, Fem buckling analysis of quasi-isotropic symmetrically laminated rectangular composite plates with a square/rectangular cutout, Journal of Mechanical Science and Technology, 27 (5) (2013) 1427-1435.

[11] M. R. Khedmati, Z. H. M. E. Nouri and M. M. Roshanali, A comparative computational investigation on the effects of randomly distributed general corrosion on the post-buckling behavior of uniaxially loaded plates, Journal of Mechanical Science and Technology, 26 (3) (2012) 767-783.

[12] R. Seifi and N. Khoda-yari, Experimental and numerical 
studies on buckling of cracked thin-plates under full and partial compression edge loading, Thin-Walled Structures, 49 (12) (2011) 1504-1516.

[13] P. K. Datta and R. L. Carlson, Buckling and vibration of a thin tensioned sheet with an elliptical hole, Experimental Mechanics, 13 (7) (1973) 280-286.

[14] S. P. Timoshenko and J. N. Goodier, Theory of elasticity, McGraw-Hill book Company (1951).

[15] D. Shaw and Y. H. Huang, Buckling behavior of a central cracked thin plate under tension, Engineering Fracture Mechanics, 35 (6) (1990) 1019-1027.

[16] R. Brighenti, Buckling of cracked thin-plates under tension or compression, Thin-Walled Structures, 43 (2) (2005) 209224.

[17] R. Brighenti, Numerical buckling analysis of compressed or tensioned cracked thin plates, Engineering Structures, 27 (2) (2005) 265-276.

[18] R. Brighenti, Buckling sensitivity analysis of cracked thin plates under membrane tension or compression loading, $\mathrm{Nu}$ clear Engineering and Design, 239 (6) (2009) 965-980.

[19] Y. Tomita and A. Shindo, Onset and growth of wrinkles in thin square plates subjected to diagonal tension, International Journal of Mechanical Sciences, 30 (12) (1988) 921931.

[20] K. Woo and C. H. Jenkins, Analysis of crease-wrinkle interaction for thin sheets, Journal of Mechanical Science and Technology, 26 (3) (2012) 905-916.

[21] T. Kremer and H. Schürmann, Buckling of tension-loaded thin-walled composite plates with cut-outs, Composites Science and Technology, 68 (1) (2008) 90-97.
[22] A. Gilabert, P. Sibillot, D. Sornette, C. Vanneste, D. Maugis and F. Muttin, Buckling instability and pattern around holes or cracks in thin plates under a tensile load, European Journal of Mechanics. A. Solids, 11 (1) (1992) 6589.

[23] R. Seifi and A. R. Kabiri, Lateral load effects on buckling of cracked plates under tensile loading, Thin-Walled Structures, 72 (2013) 37-47.

[24] G. H. Bryan, On the stability of a plane plate under thrusts in its own plane, with applications to the "buckling" of the sides of a ship, Proceedings of the London Mathematical Society, 22 (1891) 54-67.

[25] N. Friedl, F. G. Rammerstorfer and F. D. Fischer, Buckling of stretched strips, Computers \& Structures, 78 (1) (2000) 185-190.

[26] P. J. Deolasi, P. K. Datta and D. L. Prabhakara, Buckling and vibration of rectangular plates subjected to partial edge loading (compression or tension), Journal of Structural Engineering, 22 (3) (1995) 135-144. 